\title{
Development and Feasibility of SymTrak, aMulti-domain Tool for Monitoring Symptoms of Older Adults in Primary Care
}

\author{
Patrick O. Monahan, $P D^{1,2}$, Kurt Kroenke, $M D^{1,3,4}$, Christopher M. Callahan, MD ${ }^{1,4,5}$, \\ Tamilyn Bakas, PhD ${ }^{6}$, Amanda Harrawood, $B S^{5}$, Phillip Lofton, MPH' ${ }^{5}$, Danielle Frye, BS \\ Claire Draucker, $P h D^{6}$, Timothy Stump, $M S^{7}$, Debra Saliba, $M D^{7}$, James E. Galvin, $M D, M P H^{8}$, \\ Amanda Keegan, $B A^{5}$, Mary $G$. Austrom, $P h D^{9}$, and Malaz Boustani, $M D, M P H^{1,4,5}$
}

${ }^{1}$ Indiana University School of Medicine, Indianapolis, IN, USA; ${ }^{2}$ Indiana University School of Public Health, Indianapolis, IN, USA; ${ }^{3}$ VA HSR\&D Center for Health Information and Communication, Indianapolis, IN, USA; ${ }^{4}$ Regenstrief Institute, Inc., Indianapolis, IN, USA; ${ }^{5}$ ndiana University Center for Aging Research, Indianapolis, IN, USA; 'Indiana University School of Nursing, Indianapolis, IN, USA; "University of California Borun Center and Veterans Administration, Los Angeles, CA, USA; ${ }^{8}$ Charles E. Schmidt College of Medicine, Florida Atlantic University, Boca Raton, FL, USA; ${ }^{9}$ Indiana University Department of Psychiatry, Indianapolis, IN, USA.

BACKGROUND: A clinically practical, brief, user-friendly, multi-domain self-report and caregiver-report tool is needed for tracking actionable symptoms in primary care for elderly patients with multiple chronic conditions (MCCs). OBJECTIVE: Develop and assess usability, administration time, and internal reliability of SymTrak.

DESIGN AND PARTICIPANTS: Phase I: legacy instruments, content validity, analyses of existing data, focus groups (physicians, nurses, patients, informal caregivers), and Think Aloud interviews (patients, caregivers) were used to develop SymTrak. Phase II (pilot feasibility study): 81 (27 patient-caregiver dyads, 27 patients without an identified caregiver) participants were selfadministered SymTrak in clinic.

MAIN MEASURES: SymTrak and demographic questions.

KEY RESULTS: Consistent themes emerged from phase I focus groups. Ambiguous wording was corrected with Think Aloud feedback. In phase II, patients and caregivers preferred circling words instead of numbers for item response options. SymTrak self-administration completion time in clinic was brief; mean was $2.4,3.0$, and $3.3 \mathrm{~min}$ for the finalized circlingwords version, respectively, for caregivers, dyadic patients, and patients without a caregiver; and the maximum was 6.2 min for any participant. Usability questionnaire ratings were high. Cronbach's alpha for the SymTrak 23-item total score was 0.86, 0.79, and 0.81 for caregivers, dyadic patients, and patients without a caregiver, respectively.

CONCLUSIONS: SymTrak demonstrates content validity, positive qualitative findings, high perceived usability, brief self-administered completion time, and good internal reliability.

\section{Prior Presentations None.}

Electronic supplementary material The online version of this article (https://doiorg/10.1007/s11606-018-4772-4) contains supplementary material, which is available to authorized users.

Received March 1, 2018

Revised August 29, 2018

Accepted November 21, 2018

Published online March 25, 2019
KEY WORDS: primary care; psychometrics; chronic disease; aging; selfmanagement; symptoms.

J Gen Intern Med 34(6):915-22

DOI: $10.1007 / \mathrm{s} 11606-018-4772-4$

(c) Society of General Internal Medicine 2019

\section{INTRODUCTION}

Patients attending primary care clinics, especially elderly patients, often suffer from multiple chronic conditions (MCCs) such as diabetes, dementia, chronic musculoskeletal disorders, depression, and heart failure. ${ }^{1}$ Patients with MCCs have complex health care needs and they account for two thirds of US healthcare costs. ${ }^{2}$

Chronic conditions are associated with a variety of physical, functional, cognitive, behavioral, and psychological symptoms. ${ }^{3}$, ${ }^{4}$ One third of common symptoms presented in primary care do not yield a clear-cut, disease-based explanation despite causing greater distress and impairment than many asymptomatic risk factors such as hypertension and hyperlipidemia. ${ }^{5}$ Symptoms predict health care utilization and costs, health-related quality of life, work-related disability, and overall mortality. 3, 4, 6-10

The SPADE symptoms - sleep disturbance, pain, anxiety, depression, and low energy/fatigue - are the five most prevalent, chronic, disabling, and under-treated symptoms in both the general population and in clinical practice and tend to cluster together. ${ }^{11}$ These symptoms tend to co-occur (e.g., pain and depression exhibit 30-50\% co-occurrence) ${ }^{12,13}$ and often interact synergistically; furthermore, symptom clusters have a particularly adverse impact on social and physical functioning, general health, and treatment response, suggesting it may be wise to target multiple symptoms simultaneously $5,11,13,14$.

Symptoms account for over half of all US outpatient visits annually, ${ }^{9}$ and some pharmacological and non-pharmacological interventions are effective for multiple symptom categories. ${ }^{5}$ Therefore, a valid tool that simultaneously monitors prevalent, 
chronic, disabling, and under-treated actionable symptoms, while being sensitive to treatment response, is needed in primary care. A tool that tracks symptoms over time can be used to improve diagnosis, ${ }^{15}$ guide treatment, assess severity of illness, and monitor treatment response. ${ }^{3}$ In addition, up to $57 \%$ of elderly patients are accompanied by an informal caregiver during physician visits. ${ }^{16}$ Thus, assessment of health in older adults with MCCs may be enhanced by caregiver input.

Yet, there is no existing multi-domain, non-disease-specific, clinically practical tool that is brief, inexpensive, easily scored, user friendly, and developed for both self-report and caregiverreport, while also having a focus on clinically actionable symptoms. We describe here the development of SymTrak, a brief, clinically actionable, multi-domain tool, developed for monitoring self-reported or caregiver-reported symptoms across multiple diseases and chronic conditions in primary care settings.

\section{METHODS}

\section{Setting and Sample}

Patients and their self-identified informal caregiver were recruited for focus groups and Think Aloud interviews from an academic-affiliated primary care network of clinics (phase I). A separate group of dyads, as well as non-dyadic individual patients without an identified caregiver, were recruited for a pilot feasibility study to assess usability and preliminary psychometrics (phase II). The study was approved by the institutional review board and all participants provided written informed consent. Patient inclusion criteria for both phases were (1) age $\geq 65$ years, (2) $\geq$ one primary care visit in the past 12 months, (3) $\geq$ one chronic condition according to medical records, and (4) for those participants who had an identified informal caregiver; the caregiver had to be $\geq 21$ years of age and willing to participate in the study. Patient exclusion criteria were (1) permanent resident of a nursing facility or (2) had a serious mental illness such as bipolar disorder or schizophrenia. Additionally, for phase I (but not phase II), patients were excluded if they had greater than moderate cognitive impairment (Mini Mental Status Examination $[\mathrm{MMSE}]^{17}$ score $<11$ ).

\section{Phase I: Content Validity}

SymTrak was developed based on published criteria for pragmatic tools (see Table 1), ${ }^{18-20}$ legacy instruments, and input from self-management stakeholders (physicians, nurses, clinical care assistants [CCAs], patients, and caregivers). We also gained insight from analysis of existing data. ${ }^{21}$ We reviewed selected item response formats and content from existing patient-reported outcome (PRO) instruments that had potential for tracking patient-reported and caregiver-reported outcomes in primary health care practice with a focus on clinically actionable symptoms. We drew most heavily upon the following instruments: the Patient-Reported Outcomes Measurement
Table 1 Criteria Used to Select Initial Domains and Items

\begin{tabular}{|c|c|}
\hline Criterion & $\begin{array}{l}\text { Reference } \\
\text { or } \\
\text { rationale } \uparrow\end{array}$ \\
\hline Actionable & 1,2 \\
\hline $\begin{array}{l}\text { Allow self-report and proxy } \\
\text { (e.g., caregiver) report }\end{array}$ & 3 \\
\hline $\begin{array}{l}\text { Broadly applicable (not disease-specific; easily under- } \\
\text { stood; gender, age, culture sensitive) }\end{array}$ & 1,2 \\
\hline Burden is low (brief, inexpensive, easily scored) & 1,2 \\
\hline $\begin{array}{l}\text { Chronicity } \\
\text { (i.e., not self-limited/spontaneously resolving) }\end{array}$ & 4 \\
\hline $\begin{array}{l}\text { Concerning consequences (if untreated, affects the } 6 \\
\text { D's of morbidity: death, disease, disability, discomfort, } \\
\text { dissatisfaction, destitution (costs)) }\end{array}$ & 4 \\
\hline Important to stakeholders & 1,2 \\
\hline $\begin{array}{l}\text { Prevalence (commonly occurring symptom or domain } \\
\text { in primary care) }\end{array}$ & 4 \\
\hline Psychometrically strong & 1,2 \\
\hline $\begin{array}{l}\text { Sensitive to change } \\
\text { (reliable and valid for tracking) }\end{array}$ & 1,2 \\
\hline $\begin{array}{l}\text { Serve as benchmark (useful across settings and } \\
\text { populations, publically available) }\end{array}$ & 1,2 \\
\hline $\begin{array}{l}\text { Overall "Thermometer" (in addition to possible domain } \\
\text { scores, the total score should be internally reliable and } \\
\text { adequately unidimensional to be valid for reporting a } \\
\text { single score) }\end{array}$ & 4 \\
\hline Under detected or under measured in primary care & 4 \\
\hline Unlikely to cause harm & 1,2 \\
\hline $\begin{array}{l}\text { Urgencyis high (delayed treatment will cause } \\
\text { irreversible outcomes) }\end{array}$ & 4 \\
\hline
\end{tabular}

The purpose of the criteria was for developing a clinically practical tool to monitor symptoms in primary care for patients with multiple chronic conditions

†Reference or rationale: (1) recommendations from Glasgow et al. (2013), (2) recommendations from Kroenke et al. (2015), (3) recommendations from the Working Group on Health Outcomes for Older Persons with Multiple Chronic Conditions (2012), and (4) additional recommendations from our study team experts for the goal of primary care monitoring

Information System (PROMIS) ${ }^{21-23}$ 29-item Profile and Short Forms for all domains, the Somatic Symptom Scale-8 (SSS$8)^{24}$ and Patient Health Questionnaire somatic symptom module (PHQ-15) ${ }^{25,} 26$ for physical symptoms including pain locations, the depression module of the Patient Health Questionnaire (PHQ-9), ${ }^{27,} 28$ the Generalized Anxiety Disorder 7item (GAD-7) ${ }^{29}$ scale, the PEG pain scale, ${ }^{30}$ and the Health Aging Brain Care (HABC) Monitor ${ }^{31,32}$ especially for cognitive symptoms.

\section{Phase I. Stakeholder Focus Groups and Think Aloud Interviews}

Overview. Standard focus group procedures were followed; the utilized semi-structured interview guides included general questions about the usefulness, potential applications, and interpretation of SymTrak; and small groups were conducted due to interview complexity. ${ }^{33,}{ }^{34}$ Specific questions pertained to deletion or addition of domains and items, preference for item response formats, and clarity of items.

Providers. Four focus groups were held with 13 physicians (2 to 5 per group). Two focus groups were held with 13 advanced practice nurses ( 6 and 7 per group). Participants were shown 
drafts of different versions of the tool with alternative domains, items, and item response formats. Each focus group session lasted $1 \mathrm{~h}$.

Patients and Caregivers. Eight focus groups were held with 1 to 4 patients per group and eight separate focus groups were held with 1 to 4 caregivers per group, for a total of 16 dyads. In the first part of the session, patients and caregivers participated in Think Aloud interviews that lasted between 30 and $60 \mathrm{~min}$ in which a draft of SymTrak was self-administered followed by detailed probing about the tool's wording. After a 5-min break, the focus group session lasted 30 to 60 min using the interview guide.

Clinical Care Assistants. After revisions to the tool based on provider, patient, and caregiver focus groups, two Think Aloud interview sessions were held with 8 CCAs (four in each group). The CCAs were asked to identify language on SymTrak that might be difficult to understand for patients and caregivers based on their extensive experience administering established instruments in our aging brain care clinics. $^{35-38}$

\section{Phase II. Pilot Feasibility Study of Usability and Preliminary Psychometrics}

After revising SymTrak based on phase I findings, 81 participants of three types (27 patients, their 27 caregivers, and 27 patients without a caregiver) completed a brief survey, consisting of SymTrak and demographic data, in a private room in the clinics. In addition to self-administration time and internal reliability, preference for circling words instead of numbers for item responses was assessed (see Fig. 1). Specifically, two forms of SymTrak were administered back-to-back, with order randomized, and were identical except for item response format. Usability was assessed with an 8-item questionnaire after the second SymTrak administration.

\section{Analysis}

In phase I, the interviews and focus group sessions were digitally recorded, transcribed, and checked for accuracy. Standard content analysis procedures ${ }^{39}$ were used to analyze transcripts. Two raters (CD and PM) independently coded the transcripts, categorized the responses, and, through discussion, reached consensus on major themes.

For phase II, Cronbach's alpha was used to assess internal reliability. Frequency distributions did not differ significantly by participant type for usability questions or format preference (circling words versus numbers); therefore, these percentages were reported for the total sample, and the goodness-of-fit chisquare test for format preference was performed using the SAS SURVEYFREQ procedure to account for within-dyad correlation. A linear mixed model was used to analyze administration completion time from the $2 \times 2$ cross-over design; the independent variables were participant type, response format, period (1st occasion vs 2nd occasion), and order (circling words administered first vs circling numbers administered first). All tests were two-sided with alpha of 0.05 .

\section{RESULTS}

\section{Initial Selection of Domains and ltems}

We selected an initial set of domains and items based on criteria for developing clinically practical measures relevant to MCCs, ${ }^{18-20}$ as well as content validity, and review of extant instruments. We initially selected the following domains as important for tracking symptom outcomes for MCCs: physical, psychological, functional, cognitive, sleep disturbance, fatigue, and pain. Items were selected and/or adapted from publically available legacy instruments.

\section{Phase I: Think Aloud Interviews and Focus Group Results}

Characteristics of Focus Groups. The elderly patients were diverse with respect to age, education, race, income, and marital status (Table 2).

General Findings About SymTrak Purpose, Content, Administration, and Utility. Table 3 summarizes the main issues or themes that emerged. Providers preferred a monitoring tool (issue \#1) that is brief (issues \#2, \#3, and \#4). Role-specific items confused caregivers and patients who wondered whether the task is a "problem" for a patient who either does not perform the task routinely or lacks capacity to perform a task that is performed by the caregiver to the satisfaction of the dyad (issue \#5). Participants suggested we avoid these items or supply a "not applicable" box; we opted for simplicity by generally avoiding role-specific items. The exceptions were two clinically important items "Trouble taking medication in the right dose at the right time" and "Trouble remembering appointments." Providers varied in their recommendation regarding administration frequency (issue \#6) but were unanimous in requiring quick administration and readily accessible scores from medical records in graphical format (issue \#7). Another finding pertaining to utility (issue \#7) was that providers, patients, and caregivers preferred the tool to fit on one page (for printed copies) but only if such a presentation was readable with large enough font and not overly busy.

Bundled Items (Issue \#8). The rationale for bundled items (e.g., when synonyms help describe the symptom) is explained elsewhere. ${ }^{18}$ However, for items in which it is common for only one of its bundled components to be experienced (e.g., "Trouble falling asleep or trouble staying asleep," or "pain in back, arms, legs, or joints,") about $20 \%$ of 
a Circling words for the response options

PLEASE CIRCLE ONLY 1 ANSWER ON EACH ROW

\begin{tabular}{|c|c|c|c|c|c|}
\hline \multicolumn{6}{|c|}{ OVER THE PAST TWO WEEKS, HOW OFTEN HAVE YOU HAD PROBLEMS WITH: } \\
\hline 1. & FEELING TIRED OR HAVING LOW ENERGY & NeVER & SOMETIMES & OFten & AlwaYs \\
\hline 2. & TROUBLE FALLING ASLEEP OR TROUBLE STAYING ASLEEP & NEVER & SOMETIMES & Often & ALWAYS \\
\hline 3. & PAIN INTERFERING WITH DAILY ACTIVITIES & NEVER & SOMETIMES & OFten & AlwaYs \\
\hline
\end{tabular}

b Circling numbers for the response options

PLEASE CIRCLE ONLY 1 ANSWER ON EACH ROW

\begin{tabular}{|c|c|c|c|c|c|}
\hline \multicolumn{6}{|c|}{ OVER THE PAST TWO WEEKS, HOW OFTEN HAVE YOU HAD PROBLEMS WITH: } \\
\hline & & NeVER & SOMETIMES & OFTEN & ALWAYS \\
\hline 1. & FEELING TIRED OR HAVING LOW ENERGY & 0 & 1 & 2 & 3 \\
\hline 2. & TROUBLE FALLING ASLEEP OR TROUBLE STAYING ASLEEP & 0 & 1 & 2 & 3 \\
\hline 3. & PAIN INTERFERING WITH DAILY ACTIVITIES & 0 & 1 & 2 & 3 \\
\hline
\end{tabular}

Fig.1 Example of circling words versus circling numbers for item response format.

Table 2 Participant Characteristics

\begin{tabular}{|c|c|c|c|c|}
\hline \multirow[t]{2}{*}{ Characteristic } & \multirow{2}{*}{$\frac{\text { Phase I }}{\text { Patients }(N=15)}$} & \multirow{2}{*}{$\begin{array}{l}\text { Phase II } \\
\text { Patients (individual; } N=27 \text { ) }\end{array}$} & \multirow{2}{*}{$\begin{array}{l}\text { Phase II } \\
\text { Patients (dyad; } N=27 \text { ) }\end{array}$} & \multirow{2}{*}{$\begin{array}{l}\text { Phase II } \\
\text { Caregivers (dyad; } N=27)\end{array}$} \\
\hline & & & & \\
\hline Age, mean (SD);median; range & $\begin{array}{l}75.1(6.0) \\
77 ; 65-83\end{array}$ & $\begin{array}{l}72.3(7.5) \\
73 ; 51-84\end{array}$ & $\begin{array}{l}77.4(6.6) \\
77 ; 67-93\end{array}$ & $\begin{array}{l}53.4(13.2) \\
55 ; 27-72\end{array}$ \\
\hline Female sex, no. (\%) & $9(60.0)$ & $22(81.5)$ & $22(81.5)$ & $22(81.5)$ \\
\hline \multicolumn{5}{|l|}{ Race, no. (\%) } \\
\hline White & $5(33.3)$ & $14(51.9)$ & $11(40.7)$ & $9(33.3)$ \\
\hline Black & $10(66.7)$ & $13(48.1)$ & $16(59.3)$ & $15(55.6)$ \\
\hline Other & $0(0.0)$ & $0(0.0)$ & $0(0.0)$ & $3(11.1)$ \\
\hline \multicolumn{5}{|l|}{ Ethnicity, no. (\%) } \\
\hline Hispanic or Latino & $0(0.0)$ & $2(7.4)$ & $1(3.7)$ & $1(3.7)$ \\
\hline \multicolumn{5}{|c|}{ Highest level of education, no. (\%) } \\
\hline$<$ High school graduate & $5(33.3)$ & $9(33.4)$ & $17(62.9)$ & $5(18.5)$ \\
\hline High school graduate & $4(26.7)$ & $8(29.6)$ & $5(18.5)$ & $8(29.6)$ \\
\hline Some college or higher & $6(40.0)$ & $10(37.0)$ & $5(18.5)$ & $14(51.9)$ \\
\hline \multicolumn{5}{|c|}{ Total household income, past year, no. $(\%)$} \\
\hline$<\$ 15,000$ & $4(26.7)$ & $22(81.5)$ & $14(51.9)$ & $9(33.3)$ \\
\hline$\$ 15,000-\$ 30,000$ & $4(26.7)$ & $3(11.1)$ & $7(25.9)$ & $7(25.9)$ \\
\hline$>\$ 30,000$ & $2(13.3)$ & $1(3.7)$ & $4(14.8)$ & $10(37.0)$ \\
\hline Unknown & $5(33.3)$ & $1(3.7)$ & $2(7.4)$ & $1(3.7)$ \\
\hline \multicolumn{5}{|l|}{ Marital status, no. (\%) } \\
\hline Married/living together & $6(40.0)$ & $3(11.1)$ & $4(14.8)$ & $9(33.3)$ \\
\hline Widowed & $5(33.3)$ & $7(25.9)$ & $15(55.6)$ & $1(3.7)$ \\
\hline Divorced/separated & $3(20.0)$ & $15(55.6)$ & $7(25.9)$ & $5(18.5)$ \\
\hline Never married & $4(8.3)$ & $2(7.4)$ & $0(0.0)$ & $12(44.4)$ \\
\hline Unknown & $1(6.7)$ & $0(0.0)$ & $1(3.7)$ & $0(0.0)$ \\
\hline \multicolumn{5}{|l|}{ General health* } \\
\hline \multicolumn{5}{|l|}{ Physical health } \\
\hline Poor & N/D & $5(18.5)$ & $3(11.5)$ & $4(14.8)$ \\
\hline Fair & N/D & $11(40.7)$ & $15(57.7)$ & $9(33.3)$ \\
\hline Good & N/D & $8(29.6)$ & $7(26.9)$ & $10(37.0)$ \\
\hline Very good & $\mathrm{N} / \mathrm{D}$ & $2(7.4)$ & $0(0.0)$ & 3 (11.1) \\
\hline Excellent & $\mathrm{N} / \mathrm{D}$ & $1(3.7)$ & $1(3.8)$ & $1(3.7)$ \\
\hline \multicolumn{5}{|l|}{ Emotional health } \\
\hline Poor & $\mathrm{N} / \mathrm{D}$ & $0(0.0)$ & $2(7.7)$ & $0(0.0)$ \\
\hline Fair & $\mathrm{N} / \mathrm{D}$ & $11(40.7)$ & $9(34.6)$ & $12(44.4)$ \\
\hline Good & N/D & $11(40.7)$ & $14(53.9)$ & $12(44.4)$ \\
\hline Very good & N/D & $4(14.8)$ & $1(3.8)$ & $2(7.4)$ \\
\hline Excellent & $\mathrm{N} / \mathrm{D}$ & $1(3.7)$ & $0(0.0)$ & $1(3.7)$ \\
\hline
\end{tabular}

*The stem for general health items was "In general, how would you describe your["your loved one's" for caregiver-report]" physical health [and emotional health]. General health items were not collected or determined (N/D) for phase I. For phase I, demographic information was not available for caregivers, providers, or CCAs. As seen in this table, the majority of phase II participants reported fair to good physical health and emotional health 
Table 3 Phase I: Summary of Think Aloud and Focus Group Findings

\begin{tabular}{|c|c|c|}
\hline \# & Issues or themes & Key findings \\
\hline 1 & Main purpose of tool: screening vs. monitoring & $\begin{array}{l}\text { Providers felt the tool would be most useful for monitoring symptoms over time. } \\
\text { A few providers felt it might be useful to detect hidden symptoms (e.g., hearing loss) } \\
\text { or symptoms not routinely assessed. }\end{array}$ \\
\hline 2 & Somatic symptoms: brief vs. longer somatic domain & Providers preferred a briefer 11-item, instead of longer 17-item, somatic domain. \\
\hline 3 & $\begin{array}{l}\text { Somatic symptoms: single-item vs. multiple-item } \\
\text { sleep, fatigue, and pain domains }\end{array}$ & $\begin{array}{l}\text { Providers preferred a single item instead of } 3 \text { items for each of the sleep disturbance, } \\
\text { fatigue, and pain interference domains. }\end{array}$ \\
\hline 4 & Adding more items* & $\begin{array}{l}\text { No item (except poor appetite or overeating) received sufficient support from focus groups } \\
\text { to be added. }\end{array}$ \\
\hline 5 & Role-specific items & $\begin{array}{l}\text { Avoid activities that are either not done routinely or tend to be role-specific } \\
\text { (e.g., managing money, shopping, cooking, household chores). This eliminates the need for } \\
\text { a "not applicable" response. }\end{array}$ \\
\hline 6 & Frequency of administration & $\begin{array}{l}\text { Providers varied in recommendations for when and how often the tool should be administered } \\
\text { (e.g., every clinic visit, once per quarter, or adapted to patient's stability, frailty, or severity). }\end{array}$ \\
\hline 7 & Utility & $\begin{array}{l}\text { If self-administrated in clinics, providers felt } 5 \mathrm{~min} \text { is a feasible completion time. } \\
\text { Providers emphasized accessibility of scores. Incorporating scores into electronic medical } \\
\text { records would be desirable, including graphical displays to see changes over time. }\end{array}$ \\
\hline 8 & Bundled vs. split items & $\begin{array}{l}\text { Bundled items were used if synonyms provided clarity or if including related concepts in a } \\
\text { single item kept the tool short. Participants desired brief items with enough synonyms to } \\
\text { achieve intended meaning. }\end{array}$ \\
\hline 9 & $\begin{array}{l}\text { Type of item response options: frequency vs. } \\
\text { severity or quantity } \dagger\end{array}$ & $\begin{array}{l}\text { All focus group members (providers, patients, caregivers) were equally split between } \\
\text { preferences for frequency vs. severity or quantity responses. Frequency was selected for } \\
\text { several reasons (see text). }\end{array}$ \\
\hline 10 & Number of response options: 3 vs. 4 vs. 5 & $\begin{array}{l}\text { Physicians preferred } 3 \text { options with clinically meaningfully large jumps to provide } \\
\text { actionable information at the item level. Nurses, patients, and caregivers were mixed } \\
\text { (3, } 4 \text {, or } 5 \text { options). Four options were selected (see text). }\end{array}$ \\
\hline 11 & Timeframe: 1,2 , or 4 weeks & $\begin{array}{l}\text { Providers preferred } 1 \text { to } 4 \text { weeks; most felt } 2 \text { weeks was adequate. } \\
\text { The two-week timeframe was selected (See text). }\end{array}$ \\
\hline 12 & Complexity, health literacy, and readability & $\begin{array}{l}\text { Several items were reworded, based on patient and caregiver feedback, to accommodate a } \\
\text { wide range of health literacy while retaining intended construct meaning. }\end{array}$ \\
\hline
\end{tabular}

*Providers, patients, and caregivers were open-endedly asked whether they recommended any items be added to the tool. They were subsequently shown a list of additional potential items and asked whether any should be added to the tool. This list of additional potential items was initially created by our study team but was also iteratively revised between focus group sessions based on focus group feedback

Frequency options (never, rarely, sometimes, often, always) vs. severity or quantity options (not at all, a little bit, moderately, quite a bit, extremely). We investigated inserting "bother" into the introductory stem for frequency and quantity options but decided that assessing bother (as opposed to "problems with") required, for several items, too much extrospection by caregivers about the patient's perception

patients and caregivers preferred (verified by CCA focus groups) either a separate item for each component or the option to mark which component(s) were experienced. We retained bundled items, after modifications to avoid ambiguity, because patients and caregivers reported that the wording for each item needed to be "brief enough to not confuse" but also detailed enough with a small number of synonyms so that they would not have to "guess what the providers want from them."

\section{Frequency Versus Severity or Quantity Response Options} (Issue \#9). We chose frequency response options because of (1) the established practice of using frequency for psychological symptoms (e.g., PHQ-9, GAD-7, PROMIS depression and anxiety scales), (2) our goal to keep the tool and its administration simple by using only one set of item response options, and (3) our decision to use four response options (see next section) which worked well on a frequency format. Our one concern was whether the frequency format would adequately capture severity of pain (in specific locations); however, the pain interference item minimized this concern.

Number of Item Response Options (Issue \#10). Based on existing data from a randomized controlled trial to treat chronic pain ${ }^{21}$, we analyzed items with various response option sets (e.g., frequency, severity, and quantity) from legacy instruments (e.g., PROMIS 29-item Profile, PHQ-9, GAD-7) to test the reduction from five response options to four or three hypothetical response options based upon collapsing adjacent options. When compared to external validators (excellent-to-poor general health item; disability days), the reduction from five to four response options revealed a small loss of validity correlation, whereas the reduction from four to three options resulted in a substantially greater loss. To balance focus group preferences (issue \#10) with psychometric validity, we chose four response options.

Response Timeframe (Issue \#11). We chose 2 weeks as a balance between a short enough interval to recall accurately and a long enough interval to capture non-momentary states of health perceptions. In addition, the Diagnostic and Statistical Manual of Mental Disorders (DSM-V) ${ }^{40}$ uses a 2-week time frame in its diagnostic criteria for several disorders (e.g., major depressive disorders). ${ }^{41}$

Issues of Complexity, Health Literacy, and Readability (Issue \#12). A theme from all provider focus groups was to keep the tool as simple as possible. We revised items not understood by patients and caregivers during Think Aloud sessions (see Appendix 1). After these revisions, CCAs had minor additional suggestions and unanimously reported that SymTrak was easy to understand. 
Table 4 Phase II (Pilot Study): Internal Reliability and Administration Time of SymTrak

\begin{tabular}{|c|c|c|c|c|}
\hline \multirow[t]{3}{*}{ Participant type } & \multicolumn{2}{|c|}{ Cronbach's coefficient alpha } & \multicolumn{2}{|c|}{$\begin{array}{l}\text { Administration time (minutes) } \\
\text { Mean (SD); median; min-max }\end{array}$} \\
\hline & \multicolumn{2}{|l|}{ Response format } & \multicolumn{2}{|l|}{ Response format } \\
\hline & Circle wordsversion & Circle numbersversion & Circle wordsversion & Circle numbersversion \\
\hline Patients (individual) $(n=27)$ & 0.81 & 0.83 & $3.0(1.2) ; 3.0 ; 1.2-6.0$ & 2.8 (1.1); 2.7; 0.8-5.4 \\
\hline Patients (dyad) $(n=27)$ & 0.79 & 0.82 & $3.3(1.3) ; 3.1 ; 1.5-6.2$ & $3.3(1.5) ; 3.1 ; 1.4-6.8$ \\
\hline Caregivers (dyad) $(n=27)$ & 0.86 & 0.81 & $2.4(1.0) ; 2.2 ; 1.0-5.4$ & $2.3(1.0) ; 2.0 ; 1.0-4.7$ \\
\hline
\end{tabular}

\section{Phase II: Pilot Study Results}

Characteristics of Participants. Patients and caregivers in phase II were diverse with respect to race, education, income, and marital status (Table 2). Most (63\%) of the 27 caregivers were adult children, $15 \%$ were spouses, $7 \%$ grandchildren, and $15 \%$ "other." Importantly, $96 \%$ of caregivers knew the patient "very well" (not shown in Table 2).

Data Distribution and Internal Reliability. SymTrak total scores were approximately normally distributed, an advantage for parametric assumptions in statistical models. Internal reliability was good, with coefficient alpha ranging from 0.79 to 0.86 for SymTrak total score across participant types and response formats (Table 4).

Usability. Higher scores represent greater usability (Table 5). For three negatively worded items (\#1, 2, and 8), "strongly disagree" represents the best usability; 77 to $86 \%$ of patients and caregivers responded that they either strongly disagreed or disagreed with those negative statements (Table 5). For the five positively worded items, 74 to $87 \%$ of patients and caregivers responded that they "strongly agreed" or "agreed" or were "neutral" with those positive statements. Of note, $78 \%$ and $81 \%$ either strongly agreed or agreed for the two questions related to health literacy, "made sense to me" and "were easy to understand," respectively.

Preference of Circling Words Versus Circling Numbers. The observed preference distribution was $51 \%$ for circling words,
$18 \%$ for circling numbers, and $31 \%$ of patients and caregivers were neutral. This was significantly different than the theoretically expected distribution of $33.33 \%$ for each category $(p=.01)$.

\section{Self-Administration Time in Clinic Using Paper and Pencil.} The means and medians for completion time, which ranged from 2.0 to $3.3 \mathrm{~min}$, indicated quick completion regardless of participant type or response format (Table 4). Administration time was not significantly different for circling words versus circling numbers. Completion time was significantly longer (but still brief) for dyadic patients than for their caregivers (mean 3.3 vs $2.4 \mathrm{~min}$ for the circling words format, $p=0.002$ ).

Finalized SymTrak Forms. Based on positive phase II findings, no items were further revised. Because the worded response format was preferred, we used it for the finalized SymTrak Self-Report (Appendix 2) and Caregiver Report (Appendix 3) forms. These forms include two general health rating items that can be used for external validation and additional clinical information but are not counted in the 23item SymTrak total score.

\section{DISCUSSION}

After iterative revisions during focus groups and Think Aloud interviews, SymTrak was developed, and in phase II demonstrated favorable psychometric properties including an

Table 5 Phase II (Pilot Study): SymTrak Usability

\begin{tabular}{|c|c|c|c|c|c|}
\hline Please rate how you feel about SymTrak in the following 8 areas.* & Strongly disagree & Disagree & Neutral & Agree & Strongly agree \\
\hline \multicolumn{6}{|l|}{ Values are frequency (row \%) $\dagger$} \\
\hline $\begin{array}{l}\text { 1. The questions took too much time } \$(n=80) \\
\text { 2. The questions made me nervous }(n=80) \\
\text { 3. The questions were enjoyable }(n=80) \\
\text { 4. The questions were important to me }(n=80) \\
\text { 5. The questions were interesting to me }(n=78) \\
\text { 6. The questions made sense to me }(n=79) \\
\text { 7. The questions were easy to understand }(n=80) \\
\text { 8. The questions were difficult to pay attention to } \$(n=80)\end{array}$ & $\begin{array}{l}37(46.3) \\
51(63.8) \\
8(10.0) \\
6(7.5) \\
7(9.0) \\
6(7.6) \\
6(7.5) \\
40(50.0)\end{array}$ & $\begin{array}{l}29(36.3) \\
18(22.5) \\
13(16.3) \\
5(6.3) \\
5(6.4) \\
4(5.1) \\
4(5.0) \\
22(27.5)\end{array}$ & $\begin{array}{l}10(12.5) \\
7(8.8) \\
32(40.0) \\
21(26.3) \\
23(29.5) \\
7(8.9) \\
5(6.2) \\
9(11.2)\end{array}$ & $\begin{array}{l}3(3.8) \\
4(5.0) \\
20(25.0) \\
30(37.5) \\
28(35.9) \\
32(40.5) \\
32(40.0) \\
4(5.0)\end{array}$ & $\begin{array}{l}1(1.3) \\
0(0.0) \\
7(8.8) \\
18(22.5) \\
15(19.2) \\
30(38.0) \\
33(41.3) \\
5(6.3)\end{array}$ \\
\hline
\end{tabular}

*The instructions were "We would like to understand whether you think the Symptom Tracker questionnaire was easy to use"

tResponse distributions were not statistically different for the two patient samples and caregivers; therefore, it is appropriate to interpret the percentages in this table, in which all three participant types were combined

$\ddagger$ Three items are negatively worded for which "strongly disagree" is the most favorable response 
approximately normal distribution for total score and high internal reliability. The tool was also perceived by patients and caregivers as relevant, important, easy to understand, easy to complete, and enjoyable to complete without eliciting negative emotions. The maximum completion time of $6.2 \mathrm{~min}$ for any patient or caregiver was encouraging for the busy primary care setting. The tool was constructed with the flexibility and capability of being completed online or by paper and pencil.

Physicians reported that they want the ability to inspect items, domains (pending future factor analyses), and total scores of the SymTrak tool (i.e., to zoom in and out). From a practical standpoint, clinicians can handle multiple items or domains when they are familiar with a measure. For example, a comprehensive metabolic profile typically reports 4 electrolyte values, several liver function tests, creatinine, calcium, uric acid, and other serum values. The experienced clinician can quickly scan this CMP for outliers (tagged as high or low) and focus on these efficiently. The same could be done with a PRO symptom monitor like SymTrak. Clinicians might likewise focus on specific symptoms that the patient rates as most problematic on SymTrak. Alternatively, it is possible that in populations with MCCs where multiple symptoms co-occur and can derive from several conditions, the SymTrak total score may serve as an overall indicator of symptom burden and impairment (i.e., a comorbidity "thermometer"). The total score might be useful to monitor patients with MCCs for early detection of deterioration as well as to identify patients who warrant greater clinical attention. Finally, where treatment of one symptom synergistically affects response of other symptoms, ${ }^{5,11,13,14}$ strictly unidimensional subdomains may not emerge and the SymTrak total score may serve as a composite indicator of treatment outcomes.

\section{Future Investigations}

A larger-sample psychometric study of SymTrak is needed. To be valid for tracking symptoms, it will be important to establish test-retest reliability and sensitivity to change, the latter of which we are currently assessing with respect to the Health Utility Index, Mark 3 (HUI3). ${ }^{42}$ The utility of specific score thresholds for action remains to be demonstrated. Also, deployment of SymTrak in clinical practice settings is needed to evaluate and refine its optimal use. This includes the use of smartphone applications, such as the one we built, to capture its data.

\section{Limitations}

Due to concerns about clinic burden, additional instruments that could have been used for further construct validation were not administered in this pilot study. The sample size was not large enough to perform factor analysis. The present study can be generalized only to adults 65 and older. We anticipate that SymTrak will be found valid for all adults 18 and older; however, this requires future investigation.

\section{Clinical Application}

Incorporating PROs into busy practice settings will face barriers such as time limitations and workflow interruptions, but technology that reduces clinic personnel time such as automated administration and scoring (e.g., tablet computers, kiosks, and secure websites) can diminish these barriers. Home-based administration and monitoring is another strategy for minimizing disruption of clinic workflow. Access to PRO scores from electronic medical records in a graphical format would reveal time trends that can be readily discerned by busy clinicians. Clinicians could benefit from the development and validation of "red flag" thresholds for which action might be considered at the total, (potentially) domain, and item score levels. Action can include repeating or following the score, further testing, specific treatment, or referral. Based on focus groups, SymTrak has strong content validity, and has proved to be usable, efficient (quick administration time), and internally reliable..

Contributors: None.

Corresponding Author: Patrick O. Monahan, PhD; Indiana University School of Medicine, Indianapolis, IN, USA (e-mail: pmonahan@iu. edu).

Funders This work was supported by a National Institute on Aging RO1 award to P.O.M. (RO1 AG043465).

\section{Compliance with Ethical Standards:}

The study was approved by the institutional review board and all participants provided written informed consent.

Conflict of Interest: Patrick O. Monahan is a Chief Technology Officer and has 2.5\% equity ownership (valued at \$2500) in a for-profit company called RestUp.MalazBoustani is a deferred-paid advisor with 5\% equity (valued at \$5000) in the same company, RestUp. The purpose of RestUp is to use internet and mobile technology to connect caregivers and care seekers. The caregivers are paid hourly, as 1099 contractors, by care seekers, and RestUp earns its income by receiving a percentage of each hour worked. The present paper has no overlap with the RestUpcompany; the SymTrak tool developed in the paper is not used in the RestUp company; and none of the activities of the RestUp company are involved in any way with the paper or the SymTrak tool. All other authors have no financial or non-financial interests.

Disclaimer: The sponsor had no role in the following: in the study design; in the collection, analysis, and interpretation of data; in the writing of the report; or in the decision to submit the article for publication. The views expressed in this article are those of the authors and do not necessarily represent the views of the National Institute on Aging.

\section{REFERENCES}

1. Schneider KM, O'Donnell BE, Dean D. Prevalence of multiple chronic conditions in the United States' Medicare population. Health Qual Life Outcomes. 2009;7:82.

2. Leroy L, Bayliss E, Domino M, et al. The Agency for Healthcare Research and Quality Multiple Chronic Conditions Research Network: overview of research contributions and future priorities. Med Care. 2014;52 Suppl 3:S15-22.

3. Sha MC, Callahan CM, Counsell SR, Westmoreland GR, Stump TE Kroenke K. Physical symptoms as a predictor of health care use and mortality among older adults. Am J Med. 2005;118:301-6. 
4. Callahan CM, Weiner M, Counsell SR. Defining the Domain of Geriatric Medicine in an Urban Public Health System Affiliated with an Academic Medical Center. J Am Geriatr Soc. 2008;56:1802-6.

5. Kroenke $\mathbf{K}$. A practical and evidence-based approach to common symptoms: a narrative review. Ann Intern Med. 2014;16:579-86.

6. Kessler RC, Zhao S, Katz SJ, et al. Past-year use of outpatient services for psychiatric problems in the National Comorbidity Survey. Am J Psychiatry. 1999;156:115-23.

7. Korten AE, Jorm AF, Jiao Z, et al. Health, cognitive, and psychosocial factors as predictors of mortality in an elderly community sample. J Epidemiol Community Health. 1999;53:83-8.

8. Kroenke K, Spitzer R, Williams JB, et al. Physical symptoms in primary care: predictors of psychiatric disorders and functional impairment. Arch Fam Med. 1994;3:774-9.

9. Kroenke K. Studying symptoms: sampling and measurement issues. Ann Intern Med. 2001;134:844-53.

10. Kroenke K. Patients presenting with somatic complaints: epidemiology, psychiatric comorbidity and management. Int $\mathrm{J}$ Methods Psychiatr Res. 2003; 12:34-43.

11. Davis LL, Kroenke K, Monahan P, Kean J, Stump TE. The SPADE symptom cluster in primary care patients with chronic pain. Clin J Pain. 2016;32:388-93.

12. Kroenke K, Bair MJ, Damush TM, et al. Optimized antidepressant therapy and pain self-management in primary care patients with depression and musculoskeletal pain: a randomized controlled trial. JAMA. 2009;301:2099-110.

13. Kroenke K, Wu J, Bair MJ, Krebs EE, Damush TM, Tu W. Reciprocal relationship between pain and depression: a 12-month longitudinal analysis in primary care. J Pain. 2011;12:964-73.

14. Aktas A, Walsh D, Rybicki L. Symptom clusters: myth or reality? Palliat Med. 2010;24:373-85.

15. Komaroff $\mathbf{A L}$. 'Minor' illness symptoms: the magnitude of their burden and of our ignorance. Arch Intern Med. 1990;150:1586-7.

16. Adelman RD, Greene MG, Ory MG. Communication between older patients and their physicians. Clin Geriatr Med. 2000;16:1-24, vii.

17. Folstein MF, Folstein SE, McHugh PR. Mini-mental state: A practical method for grading the cognitive state of patients for the clinician. J Psychiatr Res. 1975;12:189-98.

18. Kroenke K, Monahan PO, Kean J. Pragmatic characteristics of patientreported outcome measures are important for use in clinical practice. $J$ Clin Epidemiol. 2015;68:1085-92.

19. Glasgow RE, Riley WT. Pragmatic measures: what they are and why we need them. Am J Prev Med. 2013;45:237-43.

20. Working Group on Health Outcomes for Older Persons with Multiple Chronic Conditions. Universal health outcome measures for older persons with multiple chronic conditions. J Am Geriatr Soc. 2012;60:2333-41.

21. Kroenke K, Yu Z, Wu J, Kean J, Monahan P. Operating characteristics of PROMIS 4-Item depression and anxiety scales in primary care patients with chronic pain. Pain Med. 2014; 15:1892-901.

22. Cella D, Riley W, Stone A, et al. The Patient-Reported Outcomes Measurement Information System (PROMIS) developed and tested its first wave of adult self-reported health outcome item banks: 2005-2008. J Clin Epidemiol. 2010;63:1179-94.

23. Rothrock NE, Hays RD, Spritzer K, Yount SE, Riley W, Cella D Relative to the general US population, chronic diseases are associated with poorer health-related quality of life as measured by the PatientReported Outcomes Measurement Information System (PROMIS). J Clin Epidemiol. 2010;63:1195-204.
24. Gierk B, Kohlmann S, Kroenke $\mathbf{K}$, et al. The somatic symptom scale8 (SSS-8): a brief measure of somatic symptom burden. JAMA Intern Med. 2014; 174:399-407.

25. Kroenke K, Spitzer RL, Williams JB. The PHQ-15: validity of a new measure for evaluating the severity of somatic symptoms. Psychosom Med. 2002;64:258-66.

26. Kroenke K, Spitzer RL, Williams JB, Lowe B. The Patient Health Questionnaire Somatic, Anxiety, and Depressive Symptom Scales: a systematic review. Gen Hosp Psychiatry. 2010;32:345-59.

27. Spitzer RL, Kroenke $\mathbf{K}$, Williams JBW. Validation and utility of a selfreport version of PRIME-MD: The PHQ primary care study. JAMA. 1999;282:1737-44.

28. Kroenke K, Spitzer RL, Williams JBW. The PHQ-9: Validity of a brief depression severity measure. J Gen Intern Med. 2001;16:606-13.

29. Spitzer RL, Kroenke K, Williams JB, Lowe B. A brief measure for assessing generalized anxiety disorder: the GAD-7. Arch Intern Med. 2006; 166:1092-7.

30. Krebs EE, Lorenz KA, Bair MJ, et al. Development and initial validation of the PEG, a three-item scale assessing pain intensity and interference. J Gen Intern Med. 2009;24:733-8.

31. Monahan PO, Boustani MA, Alder C, et al. Practical clinical tool to monitor dementia symptoms: the HABC-Monitor. Clin Interv Aging. 2012;7:143-57

32. Monahan PO, Alder CA, Khan BA, Stump T, Boustani MA. The Healthy Aging Brain Care (HABC) Monitor: validation of the Patient Self-Report Version of the clinical tool designed to measure and monitor cognitive, functional, and psychological health. Clin Interv Aging. 2014;5:2123-32.

33. Krueger RA. Casey MA. Focus Groups: A Practical Guide for Applied Research. 4th ed.Thousand Oaks: Sage; 2009.

34. Willis GB. Cognitive Interviewing: A Tool for Improving Questionnaire Design, Thousand Oaks: Sage Publications, Inc.; 2005.

35. Callahan CM, Boustani MA, Unverzagt FW, et al. Effectiveness of collaborative care for older adults with Alzheimer disease in primary care. JAMA. 2006;295:2148-57.

36. Callahan CM, Boustani M, Sachs GA, Hendrie HC. Integrating care for older adults with cognitive impairment. Curr Alzheimer Res. 2009;6:36874.

37. Boustani MA, Sachs GA, Alder CA, et al. Implementing innovative models of dementia care: The Healthy Aging Brain Center. Aging Ment Health. 2011;15:13-22.

38. Callahan CM, Boustani MA, Weiner M, et al. Implementing dementia care models in primary care settings: The Aging Brain Care Medical Home. Aging Ment Health. 2011;15:5-12.

39. Miles MB, Huberman AM, Saldaña J. Qualitative Data Analysis: A Methods Sourcebook. Third ed.Thousand Oaks: SAGE Publications Inc.; 2014.

40. American Psychiatric Association. Diagnostic and Statistical Manual of Mental Disorders, Fifth Edition. Arlington: Arlington, VA; 2013.

41. Lowe B, Spitzer RL, Grafe $\mathbf{K}$, et al. Comparative validity of three screening questionnaires for DSM-IV depressive disorders and physicians' diagnoses. J Affect Disord. 2004;78:131-40.

42. Horsman J, Furlong W, Feeny D, Torrance G. The Health Utilities Index (HUI): concepts, measurement properties and applications. Health Qual Life Outcomes. 2003;1:54.

Publisher's Note Springer Nature remains neutral with regard to jurisdictional claims in published maps and institutional affiliations. 